\title{
Static and dynamic properties of curved vapour-liquid interfaces by massively parallel molecular dynamics simulation
}

\author{
M. T. Horsch, S. K. Miroshnichenko, J. Vrabec*, C. W. Glass, C. Niethammer, M. \\ Bernreuther, E. A. Müller, and G. Jackson
}

\begin{abstract}
Curved fluid interfaces are investigated on the nanometre length scale by molecular dynamics simulation. Thereby, droplets surrounded by a metastable vapour phase are stabilized in the canonical ensemble. Analogous simulations are conducted for cylindrical menisci separating vapour and liquid phases under confinement in planar nanopores. Regarding the emergence of nanodroplets during nucleation, a non-equilibrium phenomenon, both the non-steady dynamics of condensation processes and stationary quantities related to supersaturated vapours are considered. Results for the truncated and shifted Lennard-Jones fluid and for mixtures of quadrupolar fluids confirm the applicability of the capillarity approximation and the classical nucleation theory.
\end{abstract}

\section{Introduction}

The influence of curvature on the properties of a nanodroplet, as opposed to an interface that is planar (on the molecular level), is hard to capture experimentally. Yet it is important for refrigeration and energy technology as well as meteorology to understand fluid interfaces with extremely high curvatures because they characterize the onset of condensation and boiling processes.

M. T. Horsch, S. K. Miroshnichenko, and J. Vrabec

Thermodynamik und Energietechnik (ThEt), Institut für Verfahrenstechnik, Universität Paderborn, Warburger Str. 100, 33098 Paderborn, Germany

M. Bernreuther, C. W. Glass, and C. Niethammer

Höchstleistungsrechenzentrum Stuttgart (HLRS), Nobelstr. 19, 70569 Stuttgart, Germany

M. T. Horsch, G. Jackson, and E. A. Müller

Molecular Systems Engineering (MSE), Centre for Process Systems Engineering, Imperial College London, South Kensington Campus, London SW7 2AZ, England

* Corresponding author: Prof. Dr.-Ing. habil. Jadran Vrabec, Universität Paderborn. E-mail: jadran.vrabec@uni-paderborn.de; phone: +49 525160 2421; fax: +49 5251603522. 
Beginning in the 1930s with the work of Verschaffelt [1], researchers became aware of the necessity of taking the internal structure of fluid interfaces into account. They increasingly looked beyond the picture of a discrete dividing surface as postulated by Gibbs [2]. In the subsequent years, this led to the theoretical work of Guggenheim [3] and Tolman [4] which, nonetheless, was mostly still based on effective radii and hence on discretization.

Today, molecular dynamics (MD) simulation provides a means of accessing the internal structure and the non-equilibrium behaviour of vapour-liquid interfaces directly, on the basis of physically sound but algebraically simple effective pair potentials. For the truncated and shifted Lennard-Jones (LJ·TS) potential [5]

$$
u(r)= \begin{cases}4 \varepsilon\left[\sigma^{12}\left(r^{-12}-r_{\mathrm{c}}^{-12}\right)+\sigma^{6}\left(r_{\mathrm{c}}^{-6}-r^{-6}\right)\right], & \text { for } r<r_{\mathrm{c}}, \\ 0, & \text { for } r \geq r_{\mathrm{c}}\end{cases}
$$

with a cutoff radius of $r_{\mathrm{c}}=2.5 \sigma$, which constitutes a reliable model for the noble gases and methane [6], fluid phase boundaries have been simulated by several groups in the recent past $[6,7,8,9,10]$. Molecular simulation is particularly suitable for investigating metastable states, cf. Fig. 1. Virial isotherms that accurately describe the conditions of fluid phase coexistence at planar or curved interfaces

$$
\frac{p}{T}=\sum_{j=1}^{5} b_{j} \rho^{j}
$$

which were correlated to the present data, are given in Tab. 1. Therein, $p$ is the pressure, $T$ is the temperature, $\rho$ is the density, and the convention $k=1$ is used. On this basis, the present work regards both equilibrium and non-equilibrium phenomena for vapour-liquid interfaces of the LJ.TS fluid. Nucleation in supersaturated vapours is considered for mixtures of quadrupolar fluids as well. For a more detailed exposition, the reader is pointed to four recent articles [10,11, 12, 13].

\section{Curved fluid interfaces in equilibrium: Theory}

The Tolman [4] approach to curved vapour-liquid interfaces is based on comparing different effective radii of a droplet, namely the Gibbs adsorption radius $R_{\rho}$, for which the interfacial excess density is zero, and the Laplace radius $R_{\mathrm{L}}$ from

$$
\gamma=\frac{1}{2} R_{\mathrm{L}}\left(p_{l}-p\right),
$$

i.e. the Laplace equation in terms of the surface tension $\gamma$ and the pressure $p_{l}$ inside a droplet containing $\iota$ molecules under equilibrium conditions. The deviation between these radii, the Tolman length

$$
\delta=R_{\rho}-R_{\mathrm{L}}
$$


Fig. 1 Isotherms in a pressure-volume diagram for the LJ.TS fluid as determined by canonical MD simulation at temperatures of $T=0.65(\nabla), 0.75(\diamond), 0.85$ $(\triangle)$, and $0.95 \varepsilon(\square)$ as well as the saturated states $(\bullet)$ according to Vrabec et al. [6] in comparison with the present fifth-order virial expansion (一), cf. Tab. 1 and Eq. (2). Results in the vicinity of the spinodal line are not shown here, and no such values were used for adjusting the virial coefficients, to ensure that only states unperturbed by nucleation were taken into account.

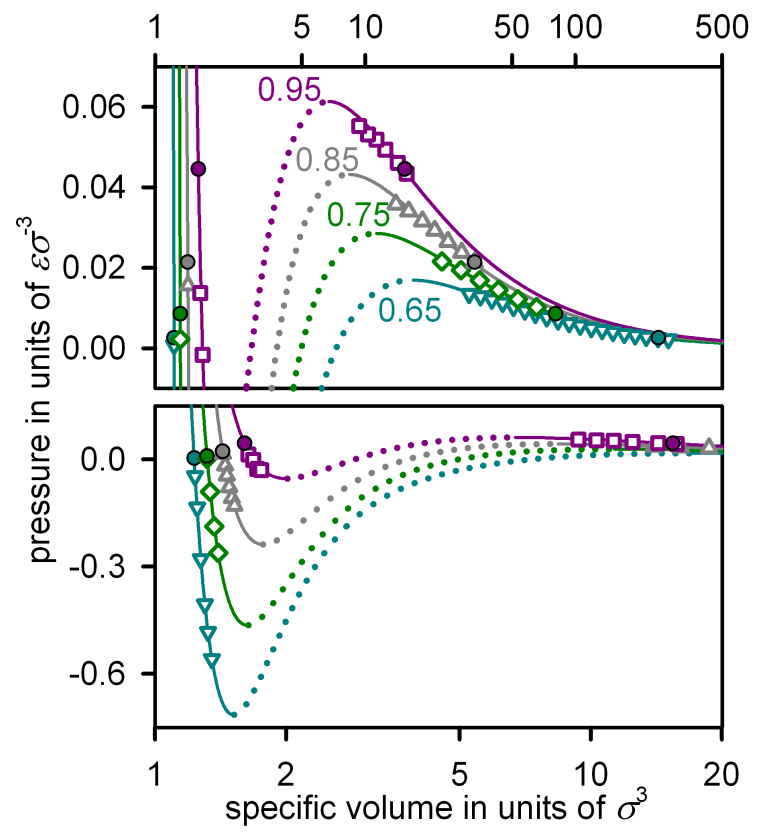

characterizes the curvature dependence of the surface tension [4]. The capillarity approximation, which postulates the droplets to be spherical and incompressible with a curvature independent surface tension, assumes $\delta$ to be zero.

Table 1 Virial coefficients for the LJ.TS fluid as determined from a fit to the MD simulation results shown in Fig. 1 and to the saturated vapour and liquid densities determined by Vrabec et al. [6]. The spinodal densities $\left(\rho^{\prime}\right)^{\#}$ and $\left(\rho^{\prime \prime}\right)^{\#}$ for liquid and vapour, respectively, were determined from the virial expansion, cf. Eq. (2).

\begin{tabular}{l||cccc|cccc}
\multicolumn{1}{c||}{$T$} & $-b_{2}$ & $b_{3}$ & $-b_{4}$ & $b_{5}$ & $\rho^{\prime}$ & $\rho^{\prime \prime}$ & $\left(\rho^{\prime}\right)^{\#}$ & $\left(\rho^{\prime \prime}\right)^{\#}$ \\
\hline 0.65 & 11.7675 & 44.5866 & 96.9625 & 71.4351 & 0.813 & 0.00406 & 0.660 & 0.0592 \\
0.7 & 9.77572 & 34.176 & 76.4866 & 59.4954 & 0.787 & 0.00728 & 0.636 & 0.0740 \\
0.75 & 8.43697 & 27.7315 & 62.373 & 50.3464 & 0.759 & 0.0124 & 0.613 & 0.0886 \\
0.8 & 7.33394 & 21.854 & 41.1349 & 40.3329 & 0.730 & 0.0198 & 0.588 & 0.103 \\
0.85 & 6.48592 & 18.3318 & 40.0252 & 34.6962 & 0.699 & 0.0304 & 0.564 & 0.119 \\
0.9 & 5.44587 & 12.3036 & 25.0989 & 23.6305 & 0.664 & 0.0446 & 0.532 & 0.134 \\
0.95 & 4.97043 & 10.0411 & 17.1387 & 16.0653 & 0.622 & 0.0648 & 0.499 & 0.149 \\
1 & 4.67665 & 9.83155 & 15.6063 & 13.8778 & 0.571 & 0.0962 & 0.466 & 0.174
\end{tabular}

According to the formalism employed by Buff [14] and Kondo [15], the surface tension becomes mimimal if it is evaluated with respect to $R_{\mathrm{L}}$. It can be shown that this assertion is only valid if the interfacial area $F$ is proportional to $R_{\mathrm{L}}^{2}$. However, both mechanical and thermodynamic equilibrium conditions for a droplet containing $\iota$ molecules imply 


$$
R_{\mathrm{L}}=2\left(\frac{\partial V_{l}}{\partial F}\right)_{N, V, T},
$$

where $V_{l}$ and $V$ are the volumes occupied by the droplet and the system as a whole, respectively. This only agrees with $F \sim R_{\mathrm{L}}^{2}$ if curvature effects cancel out.

For cylindrical interfaces, the surface tension varies with the radius according to

$$
\left[\left(\frac{\partial \ln R_{\mathrm{L}}}{\partial \ln \gamma}\right)_{T}-1\right]^{-1}=\frac{\delta}{R_{\mathrm{L}}}+\frac{\delta^{2}}{2 R_{\mathrm{L}}^{2}}
$$

an expression that is similar and analogous to Tolman's equation for droplets, where $R_{\mathrm{L}}$ is defined to be positive for convex and negative for concave menisci. By combining the Young equation [16] with an expansion of $\gamma$ to first order in $1 / R_{\mathrm{L}}$, the contact angle $\vartheta$ of a fluid confined in a planar nanopore is obtained as

$$
\cos \vartheta=\left(\frac{\gamma_{\infty}}{\Delta \gamma_{\mathrm{s}}}+\frac{\delta_{\infty}}{R_{\min }}\right)^{-1} .
$$

Therein, $\gamma_{\infty}$ is the surface tension of the planar vapour-liquid interface, $\delta_{\infty}$ is the Tolman length in the planar limit, and the pore diameter is $2 R_{\min }$, while $\Delta \gamma_{\mathrm{s}}$ indicates the difference between the specific surface energies of the substrate when it is in contact with the vapour and the liquid, respectively.

\section{Curved fluid interfaces in equilibrium: MD simulation}

Using the $l s 1$ mardyn MD program [19], equilibrium states involving droplets and cylindrical menisci were simulated for the LJ.TS fluid.

Vapour-droplet equilibrium MD simulations were conducted for droplets containing on the order of 100 to 1000 molecules in the canonical ensemble, where such equilibria can be stable - as opposed e.g. to the grand canonical ensemble where this corresponds to a free energy maximum. The droplet size was evaluated according to a version of the cluster criterion of ten Wolde and Frenkel [20] with the connectivity radius $R_{\ell}=1.5 \sigma$ and coordination numbers $j \geq 4$ defining the liquid phase. The present results correspond to moderately supersaturated vapours, cf. Fig. 2, and are consistent with the results of Vrabec et al. [6] on larger droplets as well as the study of Napari et al. [17] covering vapours at pressures that approach the spinodal line. In the intermediate regime, the droplet size in equilibrium generally agrees well with the capillarity approximation.

Cylindrical interfaces were investigated by simulating liquid slabs, cf. Fig. 4, confined between two planar and layered walls represented by coupled harmonic oscillators. The equilibrium positions of the wall atoms were aligned according to a hexagonal structure with an interatomic distance of $0.3816 \sigma$, corresponding to the bond length in graphite expressed in terms of the $\sigma$ parameter value for fluid methane. Both the fluid-fluid and the fluid-wall interactions were modelled by the 
LJ.TS potential, employing equal size parameters $\sigma_{\mathrm{fw}}=\sigma$ in both cases, while the dispersive energy between fluid molecules and wall atoms

$$
\varepsilon_{\mathrm{fw}}=\zeta \varepsilon
$$

was systematically varied. The arithmetic mean of the saturated vapour and liquid densities was selected as a criterion for detecting the phase boundary. A circle was adjusted to the resulting profile at distances between 2 and $11 \sigma$ from the wall, cf. Fig. 4, and the tangent to this circle at a distance of $1 \sigma$ from the wall was examined to determine the contact angle. Qualitatively, the contact angles obtained by the present MD simulations are captured by Eq. (7), assuming a proportionality law for

$$
\Delta \gamma_{\mathrm{s}}=\mathrm{K}_{\gamma}\left(\rho^{\prime}-\rho^{\prime \prime}\right)\left(\zeta-\zeta_{\mathrm{o}}\right),
$$

cf. Fig. 5. The magnitude of the fluid-wall dispersion for which $\vartheta$ becomes rectangular $\left(\zeta_{0}=0.118\right)$ was found to be temperature independent.

\section{Homogeneous vapour to liquid nucleation: Theory}

The foundations of the classical nucleation theory (CNT), concerning the first step of a first-order phase transition in the bulk of a metastable phase, were laid by

Fig. 2 Droplet size $\imath^{\star}$ over the supersaturation ratio $S_{\mu}$ (in terms of the chemical potential) for vapour-droplet equilibria of the LJ.TS fluid $(\bullet)$ from the present work,

$(\nabla)$ according to Vrabec et al. $[6],(\triangle)$ according to Napari et al. [17], and following the capillarity approximation $(-)$ as well as the Laaksonen et al. [18] model (- -).

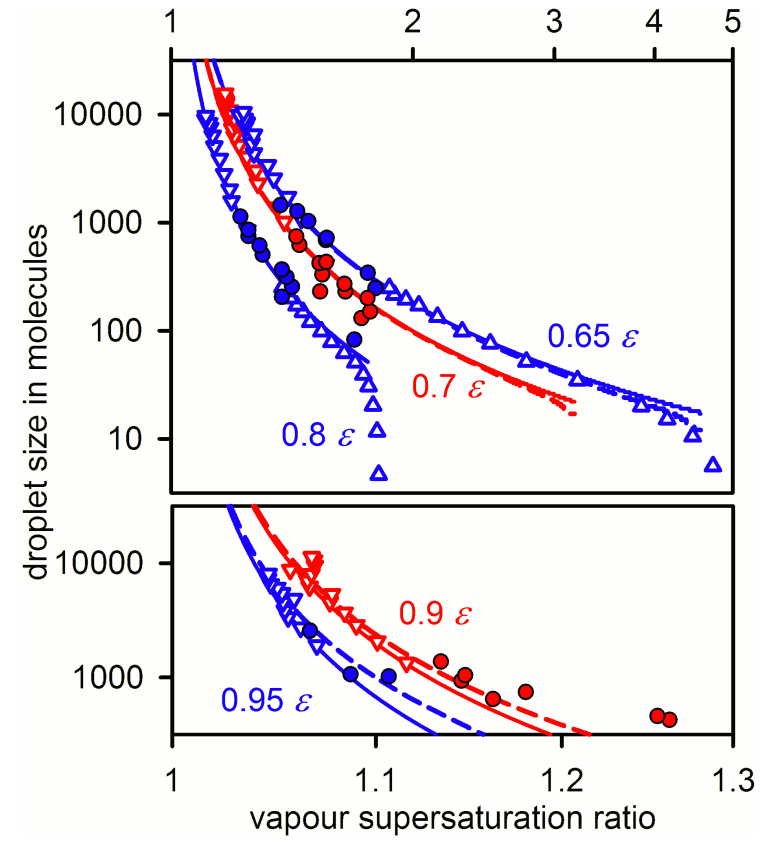


Fig. 3 Simulation snapshots for the reduced fluid-wall dispersive energy $\zeta$ of 0.09 (left) and 0.16 (right) at a temperature of $0.73 \varepsilon$. The upper half is reproduced in the bottom to illustrate the effect of the periodic boundary condition.

Fig. 4 Vapour-liquid interface profiles for the reduced fluidwall dispersive energy $\zeta$ of $0.07(\triangle), 0.10(\square), 0.13(\circ)$, and $0.16(\nabla)$ at a temperature of $0.82 \varepsilon$.

Fig. 5 MD simulation results for $\vartheta$ over $T$ with a reduced fluid-wall dispersive energy of $\zeta=0.07$ ( $), 0.09$ (०), 0.1 $(\mathbf{\nabla}), 0.11(\square), 0.13(\mathbf{\square}), 0.14$ $(\triangle)$, and $0.16(\bullet)$ as well as following the proportionality law (-, - ), cf. Eq. (7), with $\zeta_{0}=0.118, \delta_{\infty}(T)$ from a correlation based on the data of Vrabec et al. [6], and a reduced fluid-wall surface energy difference of $\mathrm{K}_{\gamma}=7$ $\sigma \varepsilon$, cf. Eq. (9).
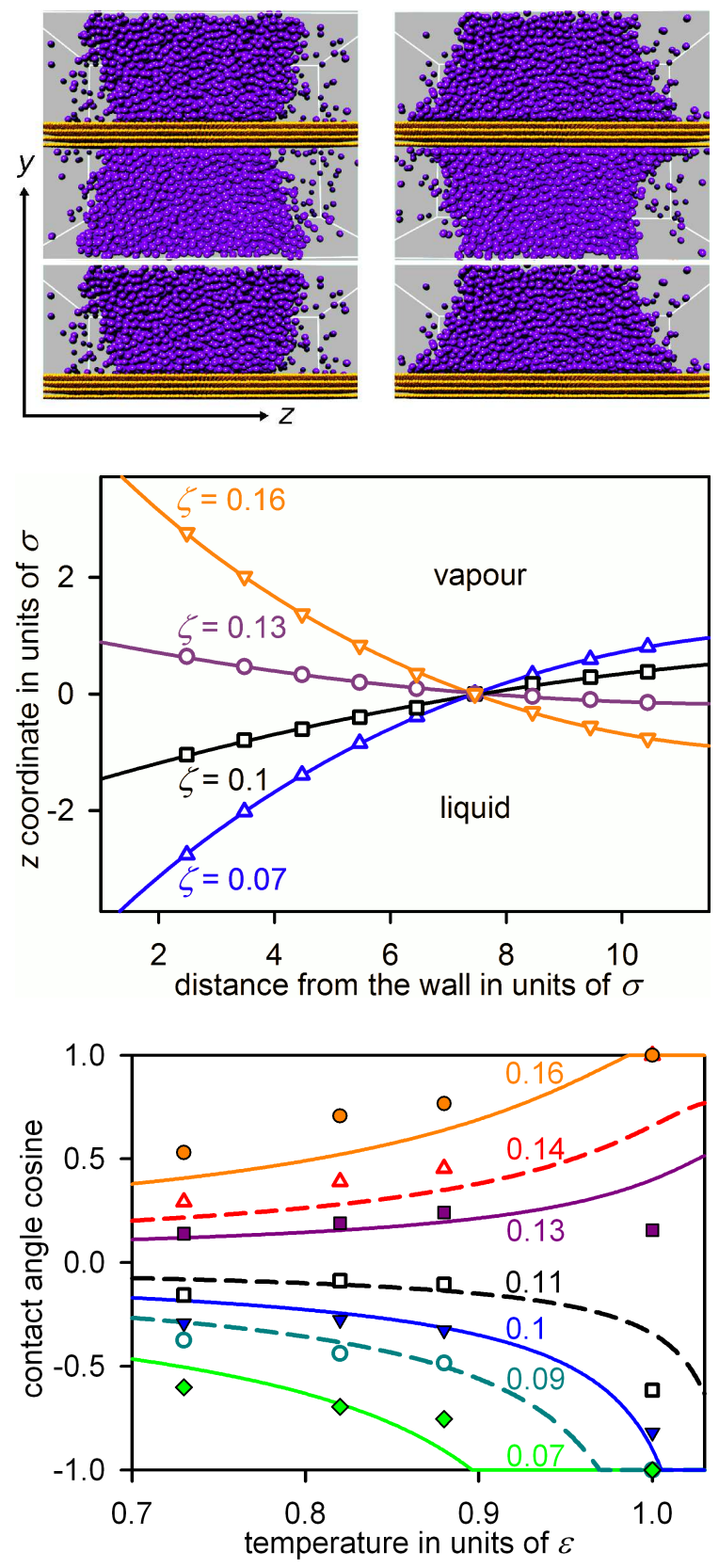

Volmer and Weber [21] as well as Farkas [22]. On the basis of the capillarity approximation, the free energy of formation $\Delta A$ of a droplet containing $l$ molecules in the thermodynamic limit (i.e. for an infinitely large vapour phase at a constant 
supersaturation ratio) evaluates to

$$
\frac{\partial A}{\partial \imath}=\gamma_{\infty} \frac{\partial F}{\partial \imath}-\left(\mu-\mu_{\iota}+\frac{p_{l}-p}{\rho^{\prime}}\right),
$$

in differential terms, where $\mu_{l}$ and $p_{l}$ are the chemical potential and the pressure inside the droplet, respectively, while $\mu$ and $p$ refer to the vapour. In the $l \rightarrow \infty$ limit, the rightmost term of Eq. (10) yields an effective chemical potential difference

$$
\Delta \mu_{\mathrm{e}}=\mu-\mu_{\mathrm{s}}(T)+\frac{p_{\mathrm{s}}(T)-p}{\rho^{\prime}},
$$

that accounts for the 'pressure effect' [23] of the vapour - which may include the contribution of an inert carrier gas. Note that $\mu_{\mathrm{s}}(T)$ and $p_{\mathrm{S}}(T)$ do not depend on $l$ since these quantities characterize the saturated bulk fluid. As visualized in Fig. 6 , the presence of a carrier gas increases the free energy barrier $\Delta A^{\star}$ of a nucleation process, i.e. the maximum of $\Delta A$ reached for a critical droplet in (unstable) equilibrium with the vapour, corresponding to the conditions discussed above.

From the analysis of a random walk over $l$, the probability for a droplet containing $\ell$ molecules to eventually reach macroscopic size can be determined as

$$
\mathrm{Q}(\ell)=\frac{\int_{1}^{\ell} \exp (2 \Delta A / T) d \iota}{\int_{1}^{\infty} \exp (2 \Delta A / T) d \iota},
$$

while the nucleation rate, i.e. the number of macroscopic liquid drops formed by homogeneous nucleation per volume and time unit, is

$$
J=\mathrm{CNT} \cdot \frac{N^{\prime} z F^{\star}}{V} \exp \left(\frac{-\Delta A^{\star}}{T}\right),
$$

Fig. 6 Free energy of formation according to CNT for $\mathrm{CO}_{2}$ droplets in supersaturated vapours at $T=250.2 \mathrm{~K}$ with $S_{\rho}=2.72$ and $\mathrm{CO}_{2}$ mole fractions of $y_{0}=1(-), 1 / 2$ $(--)$, and $1 / 3(\cdots)$, reaching a maximum for the critical droplet $(\square)$. Note that in the thermodynamic limit, i.e. in a macroscopic system, the Gibbs, Helmholtz, and Landau free energies of formation for small droplets converge.

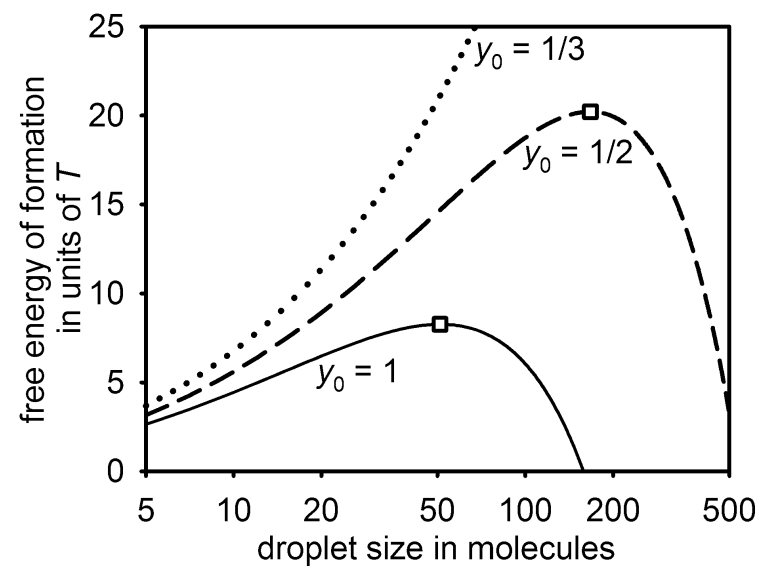


Fig. 7 Number of droplets per volume over simulation time for droplets containing $l>10$, 25, 50, and 100 molecules in a canonical ensemble MD simulation of the LJ.TS fluid at $T=0.7 \varepsilon$ and $\rho=0.03421$ $\sigma^{-3}$ in comparison with the aggregated number of dæmon interventions per volume in a grand canonical MD simulation with $T=0.7 \varepsilon, S_{\mu}$ $=2.8658$, and $\ell=51$.

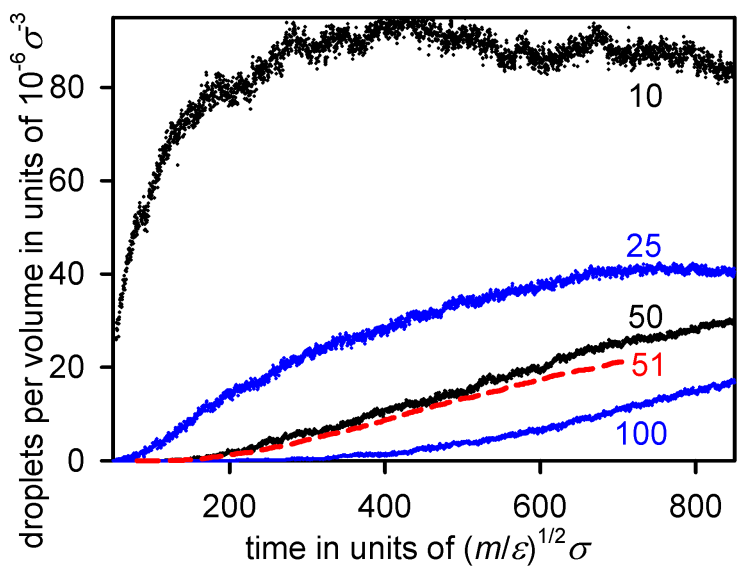

according to CNT. In this expression, $\mathrm{T}$ refers to the rate at which vapour monomers collide with an interface (per surface area), $N^{\prime}$ is the number of monomers in the system, $F^{\star}$ is the surface area of a critical droplet, and $V$ is the system volume. The correction factors C, N, and $z$ were introduced by Farkas [22], Feder et al. [24], and Zel'dovič [25], respectively. The overall pressure effect on $J$ following Eq. (13), as discussed by Wedekind et al. [23], defines the carrier gas correction factor W.

\section{Homogeneous vapour to liquid nucleation: MD simulation}

Nucleation in supersaturated vapours was studied by simulating systems containing between 100000 and 17000000 molecules, exploiting the excellent scalability of the $l s 1$ mardyn program on massively parallel computing platforms [26].

The method of Yasuoka and Matsumoto [27], where droplet formation rates are evaluated during the stage of a condensation process that corresponds to nucleation (rather than relaxation or droplet growth), was applied to the canonical ensemble. In these simulations, the vapour pressure decreased over time due to the transfer of molecules from the vapour to the dispersed liquid phase. Furthermore, steady state quantities, pertaining to nucleation only, were investigated with a new simulation method. This method combines the grand canonical ensemble with McDonald's damon [28], an intelligent being whose interventions eliminate droplets containing more than $\ell$ molecules; see Fig. 7 for a comparison between these approaches. Results for the LJ.TS fluid agree well with CNT, using a temperature independent value for the empirical correction factor $\mathrm{C}$ introduced by Farkas [22].

Canonical ensemble MD simulations were also conducted for multi-component systems containing nitrogen, oxygen, and argon - at the ratio prevalent in the earth's atmosphere - as well as carbon dioxide with a greater partial density than at saturation. The molecular models employed for this purpose, introduced by Vrabec et 
Fig. 8 Nucleation rate of the LJ.TS fluid over supersaturation from the present MD simulations of the grand canonical ensemble with McDonald's dæmon $(\bullet)$ as well as according to $\mathrm{CNT}$ with $\mathrm{C}=200(-)$ and the Laaksonen et al. [18] model (--) at temperatures of $T=0.45$, $0.65,0.7,0.85,0.9$, and 0.95 $\varepsilon$. The supersaturation ratio is given in terms of the chemical potential of the vapour phase.

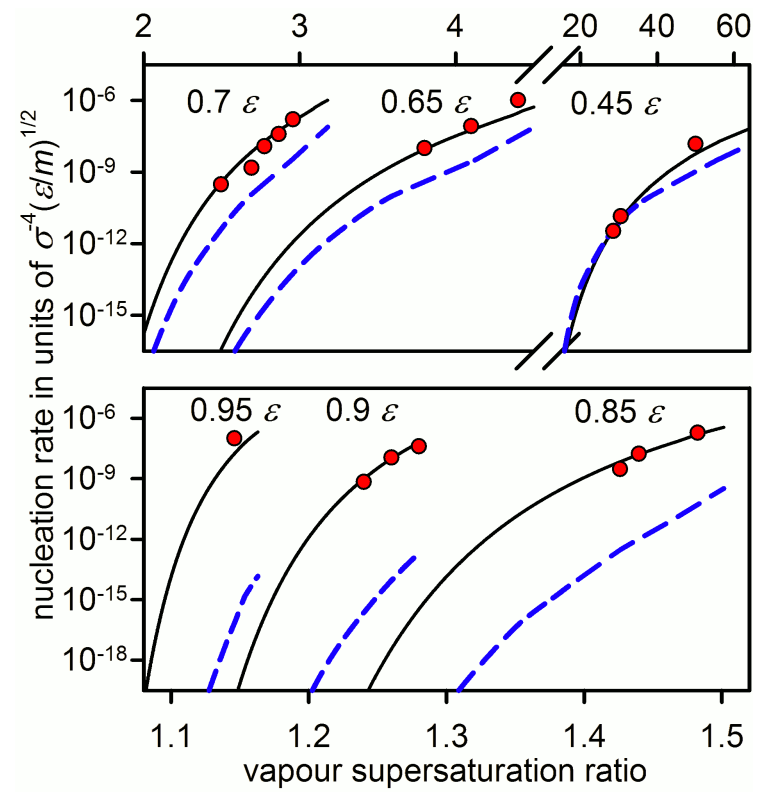

al. [29], are well-established with respect to fluid phase equilibria [29, 30, 31]. For these systems, the analysis of the carrier gas effect according to Wedekind et al. [23] is confirmed qualitatively by the determined droplet formation rates $J_{\ell}$, given in Tab. 2 , although significant quantitative deviations are present at high temperatures.

\section{Conclusion}

From the preceding analysis of curved vapour-liquid interfaces and homogeneous nucleation it can be concluded that CNT is able to capture both the nucleation rate and the critical droplet size for the considered systems, i.e. the LJ.TS fluid and a quaternary mixture of quadrupolar and unpolar fluids. The main criticism usually made of CNT is that it applies the capillarity approximation to small droplets where significant curvature effects should be expected. However, a deviation from capillarity is implicit in the prefactor $C$ which empirically accounts for its overall influence on the nucleation rate. This corresponds to stating that the capillarity approximation overestimates the free energy barrier by $T \ln C$.

The physical foundation of this approach is more robust than it might seem at first sight. By combining recent simulation results on the equilibrium vapour pressure of droplets, cf. Fig. 2, it becomes apparent that curvature effects are significant in the immediate vicinity of the spinodal line for the vapour, corresponding to $l^{\star}<100$, while they are virtually undetectable for droplets containing more than 1 000 molecules. Thus, the deviation from Eq. (10) regarding the magnitude of $\Delta A^{\star}$ 
Table 2 Droplet formation rate from Yasuoka-Matsumoto (YM) canonical ensemble MD simulation as well as critical droplet size (in molecules), Wedekind factor $\mathrm{W}$, and the prediction $J / Q_{\mathrm{CNT}}(\ell)$ for the droplet formation rate according to CNT with $C=1$, in dependence of temperature (in units of $\mathrm{K}$ ), supersaturation ratio (with respect to the partial density of carbon dioxide) and $\mathrm{YM}$ threshold size $\ell$ (in molecules) for the quaternary system $\mathrm{CO}_{2}+\mathrm{N}_{2}+\mathrm{O}_{2}+$ Ar. The mole fraction $y_{0}$ of carbon dioxide in the supersaturated vapour is indicated in the table, while the composition regarding the other fluids corresponds to the earth's atmosphere. The rates are given in units of $\mathrm{m}^{-3} \mathrm{~s}^{-1}$ and where no nucleation was detected, $J_{\mathrm{CNT}}$ instead of $J / \mathrm{Q}_{\mathrm{CNT}}$ is shown in the last column.

\begin{tabular}{|c|c|c|c|c|c|c|}
\hline$T$ & $\rho / \rho^{\prime \prime}$ & $y_{0}$ & $\ell$ & $J_{\ell}$ & $l^{\star} \mathrm{W}$ & $J / Q_{\mathrm{CN}^{-}}$ \\
\hline \multirow[t]{13}{*}{238.4} & \multirow[t]{4}{*}{2.80} & \multirow[t]{2}{*}{$1 / 2$} & 50 & $1.5 \cdot 10^{33}$ & \multirow[t]{2}{*}{660.03} & $2.6 \cdot 10^{31}$ \\
\hline & & & 85 & $1.6 \cdot 10^{32}$ & & $3.3 \cdot 10^{30}$ \\
\hline & & \multirow[t]{2}{*}{1} & 50 & $5.6 \cdot 10^{32}$ & \multirow[t]{2}{*}{411} & $9.9 \cdot 10^{31}$ \\
\hline & & & 85 & $2.1 \cdot 10^{32}$ & & $7.6 \cdot 10^{31}$ \\
\hline & \multirow[t]{4}{*}{3.08} & $1 / 2$ & 50 & $5.5 \cdot 10^{33}$ & \multirow[t]{2}{*}{650.02} & $3.1 \cdot 10^{31}$ \\
\hline & & & 150 & $3.1 \cdot 10^{32}$ & & $3.9 \cdot 10^{30}$ \\
\hline & & 1 & 50 & $6.3 \cdot 10^{33}$ & \multirow[t]{2}{*}{391} & $1.6 \cdot 10^{32}$ \\
\hline & & & 150 & $2.9 \cdot 10^{32}$ & & $1.3 \cdot 10^{32}$ \\
\hline & \multirow[t]{5}{*}{3.36} & $1 / 3$ & - & $\ll 10^{31}$ & $1274.2 \cdot 10^{-6}$ & $1.1 \cdot 10^{27}$ \\
\hline & & $1 / 2$ & 50 & $1.1 \cdot 10^{34}$ & \multirow[t]{2}{*}{650.02} & $8.7 \cdot 10^{30}$ \\
\hline & & & 300 & $3.2 \cdot 10^{32}$ & & $4.2 \cdot 10^{30}$ \\
\hline & & 1 & 50 & $6.7 \cdot 10^{33}$ & \multirow[t]{2}{*}{371} & $2.1 \cdot 10^{32}$ \\
\hline & & & 300 & $1.4 \cdot 10^{33}$ & & $1.8 \cdot 10^{32}$ \\
\hline \multirow[t]{13}{*}{$\overline{250.2}$} & \multirow[t]{4}{*}{2.34} & $1 / 2$ & 50 & $1.1 \cdot 10^{34}$ & \multirow[t]{2}{*}{$1401.9 \cdot 10^{-4}$} & $1.8 \cdot 10^{33}$ \\
\hline & & & 100 & $1.1 \cdot 10^{33}$ & & $7.8 \cdot 10^{29}$ \\
\hline & & 1 & 50 & $1.3 \cdot 10^{33}$ & \multirow[t]{2}{*}{541} & $3.9 \cdot 10^{32}$ \\
\hline & & & 100 & $3.4 \cdot 10^{32}$ & & $1.4 \cdot 10^{32}$ \\
\hline & \multirow[t]{4}{*}{2.53} & $1 / 2$ & 85 & $7.4 \cdot 10^{33}$ & \multirow[t]{2}{*}{$1431.0 \cdot 10^{-4}$} & $3.9 \cdot 10^{30}$ \\
\hline & & & 200 & $7.4 \cdot 10^{32}$ & & $3.1 \cdot 10^{28}$ \\
\hline & & 1 & 85 & $2.2 \cdot 10^{33}$ & \multirow[t]{2}{*}{521} & $1.9 \cdot 10^{32}$ \\
\hline & & & 200 & $7.7 \cdot 10^{32}$ & & $1.9 \cdot 10^{32}$ \\
\hline & \multirow[t]{5}{*}{2.72} & $1 / 3$ & - & $\ll 10^{31}$ & $8794.3 \cdot 10^{-25}$ & $2.3 \cdot 10^{8}$ \\
\hline & & $1 / 2$ & 75 & $1.3 \cdot 10^{34}$ & \multirow[t]{2}{*}{$1504.2 \cdot 10^{-5}$} & $1.8 \cdot 10^{31}$ \\
\hline & & & 250 & $1.6 \cdot 10^{33}$ & & $1.7 \cdot 10^{28}$ \\
\hline & & 1 & 75 & $4.8 \cdot 10^{33}$ & \multirow[t]{2}{*}{501} & $2.6 \cdot 10^{32}$ \\
\hline & & & 250 & $1.4 \cdot 10^{33}$ & & $2.5 \cdot 10^{32}$ \\
\hline
\end{tabular}

is dominated by an integral over the free energy of formation for extremely small droplets. At supersaturation ratios sufficiently distant from spinodal conditions, this contribution does not depend on $S$ and can be represented by $-T \ln C$ with a constant value of $C$.

Acknowledgement. The authors would like to thank J. Harting, H. Hasse, E. Y. Kenig, and G. Reina for their support and for valuable discussions. The present work, which contributes to the BMBF project IMEMO, was conducted under the auspices of the Boltzmann-Zuse Society of Computational Molecular Engineering (BZS). Major aspects of it were facilitated by the reliable technical assistance of M. Heitzig and Z. Lin. The position of M. T. Horsch at the Imperial College London is funded by the DAAD postdoc programme, and computations were performed at the 
High Performance Computing Center Stuttgart (HLRS) with resources assigned to the grant MMHBF.

\section{References}

1. J.E. Verschaffelt, Bulletin de l'Académie Royale de Belgique: Classe de Sciences 22(4), 373 (1936)

2. J.W. Gibbs, American Journal of Science: Series 3 16, 441 (1878)

3. E.A. Guggenheim, Transactions of the Faraday Society 35, 397 (1940)

4. R.C. Tolman, Journal of Chemical Physics 17(3), 333 (1949)

5. M.P. Allen, D.J. Tildesley, Computer Simulation of Liquids (Clarendon, Oxford, 1987)

6. J. Vrabec, G.K. Kedia, G. Fuchs, H. Hasse, Molecular Physics 104(9), 1509 (2006)

7. R. Hołyst, M. Litniewski, Physical Review Letters 100, 055701 (2008)

8. J.A. van Meel, A.J. Page, R.P. Sear, D. Frenkel, Journal of Chemical Physics 129, 204505 (2008)

9. B.J. Block, S.K. Das, M. Oettel, P. Virnau, K. Binder, Journal of Chemical Physics 133, $154702(2010)$

10. M. Horsch, J. Vrabec, H. Hasse, Physical Review E 78, 011603 (2008)

11. M. Horsch, J. Vrabec, Journal of Chemical Physics 131, 184104 (2009)

12. M. Horsch, M. Heitzig, C. Dan, J. Harting, H. Hasse, J. Vrabec, Langmuir 26(13), 10913 (2010)

13. M. Horsch, Z. Lin, T. Windmann, H. Hasse, J. Vrabec, Atmospheric Research (2010). In press, doi:10.1016/j.atmosres.2010.10.016, arXiv:1001.1857 [physics.ao-ph]

14. F.P. Buff, Journal of Chemical Physics 23(3), 419 (1955)

15. S. Kondo, Journal of Chemical Physics 25(4), 662 (1956)

16. T. Young, Philosophical Transactions of the Royal Society 95, 65 (1805)

17. I. Napari, J. Julin, H. Vehkamäki, Journal of Chemical Physics 131, 244511 (2009)

18. A. Laaksonen, I.J. Ford, M. Kulmala, Physical Review E 49(6), 5517 (1994)

19. M. Bernreuther, J. Vrabec, in High Performance Computing on Vector Systems, ed. by M. Resch, et al. (Heidelberg: Springer, 2006), pp. 187-195. ISBN 3-540-29124-5

20. P.R. ten Wolde, D. Frenkel, Journal of Chemical Physics 109, 9901 (1998)

21. M. Volmer, A. Weber, Zeitschrift für physikalische Chemie (Leipzig) 119, 277 (1926)

22. L. Farkas, Zeitschrift für physikalische Chemie (Leipzig) 125, 236 (1927)

23. J. Wedekind, A.P. Hyvärinen, D. Brus, D. Reguera, Physical Review Letters 101, 125703 (2008)

24. J. Feder, K.C. Russell, J. Lothe, G.M. Pound, Advances in Physics 15(1), 111 (1966)

25. Â.B. Zel'dovič, Žurnal Èksperimental'noj i Teoretičeskoj Fiziki 12, 525 (1942)

26. M. Buchholz, H.J. Bungartz, J. Vrabec, Journal of Computational Science, submitted (2010)

27. K. Yasuoka, M. Matsumoto, Journal of Chemical Physics 109(19), 8451 (1998)

28. J.E. McDonald, American Journal of Physics 31, 31 (1963)

29. J. Vrabec, J. Stoll, H. Hasse, Journal of Physical Chemistry B 105(48), 12126 (2001)

30. Y.L. Huang, J. Vrabec, H. Hasse, Fluid Phase Equilibria 287(1), 62 (2009)

31. J. Vrabec, G.K. Kedia, U. Buchhauser, R. Meyer-Pitroff, H. Hasse, Cryogenics 49, 72 (2009) 